\title{
Low-interferences Determination of the Antioxidant Capacity in Fruits Juices Based on Xanthine Oxidase and Mediated Amperometric Measurements in the Reduction Mode
}

\author{
Madalina-Petruta Bucur, Maria-Cristina RAdulescu, Bogdan Bucur, ${ }^{\dagger}$ and Gabriel Lucian RAdu \\ Centre of Bioanalysis, National Institute of Research and Development for Biological Sciences, 296, \\ Splaiul Independentei, Bucharest 060031, Romania
}

\begin{abstract}
A low-interferences enzymatic sensor for evaluating the antioxidant capacity was developed. Xanthine oxidase was used to produce superoxide radicals that spontaneously dismutate to hydrogen peroxide. Low xanthine concentrations were used to minimize the rapid dismutation of the superoxide radical before its fast reaction with antioxidants. The sensor operates in the reduction mode, and evaluations with low interferences of the antioxidant capacity are based on the detection of remaining hydrogen peroxide using Prussian blue electrodes at low potentials. The linear calibration graph is between $2-10 \mu \mathrm{M}$ ascorbic acid. No interferences were observed from easily oxidisable substances including uric acid, which is produced in the enzymatic reaction or other substances usually found in foods. The method was used to evaluate the antioxidant capacity in different real juice samples.
\end{abstract}

Keywords Antioxidant capacity evaluation, xanthine oxidase, Prussian blue, ascorbic acid

(Received July 26, 2015; Accepted August 11, 2015; Published February 10, 2016)

\section{Introduction}

Free reactive radicals are intermediates of normal cells and body metabolism, ${ }^{1}$ which have important role in signalling processes. ${ }^{2}$ However, their presence in excessive concentrations may induce oxidative stress and is associated with various diseases. ${ }^{3}$ The free radicals level is naturally controlled by endogenous enzymes (i.e. superoxide dismutase, catalase and peroxidase) and exogenous dietary antioxidants (i.e. polyphenols, vitamins $\mathrm{C}$ and E, carotenoids). ${ }^{4}$ The antioxidants intake was correlated with an improvement of the antioxidant markers level in biological fluids, ${ }^{5}$ but the physiological relevance of the antioxidant consumption was not sufficiently demonstrated according to European Food Safety Agency ${ }^{6}$ and United States Department of Agriculture (USDA). ${ }^{7}$ Numerous cosmetic products, ${ }^{8}$ food additives, ${ }^{9}$ herbal health products ${ }^{10}$ or nutritional supplements ${ }^{11}$ are marketed and commercialized based on their antioxidant content. New substances with higher antioxidant capacity are synthesized from natural compounds. ${ }^{12}$ Evaluations of the antioxidant capacity in physiological mimicking conditions are important for consumers because the real health benefits of antioxidants are being debated by scientists. ${ }^{13}$ Thus, antioxidant vitamin supplementation has no effect on the reduction of major cardiovascular events, ${ }^{14}$ or even increases the cancer risk. ${ }^{15}$

Appropriate evaluations of the antioxidant capacity of various food products are difficult, and should not only quantify the chemical composition of each sample, such as flavonoids and

$\dagger$ To whom correspondence should be addressed.

E-mail: bucurica@yahoo.com phenolic compounds detected by HPLC. ${ }^{16}$ A more physiologically relevant alternative is to quantify the antioxidant capacity by the sample interaction with different reactive oxygen species (ROS) that are generated in situ (using enzymatic or chemical reactions $)^{17}$ or commercially available free radicals (such as ABTS or DPPH ${ }^{18}$ ). The critical factors that affect the reliability of any antioxidant capacity estimation are the generation of unstable ROS and quantification of the analytical signal. One important ROS is the superoxide radical $\left(\mathrm{O}_{2^{-}}{ }^{-}\right)$, which can be produced in vitro using xanthine oxidase (XOD). The reaction catalyzed by XOD converts (hypo)xanthine to uric acid, and produces $\mathrm{O}_{2}{ }^{--}$together with hydrogen peroxide either directly (a small part) or from the dismutation of $\mathrm{O}_{2}{ }^{-{ }^{-}}$(Fig. 1). ${ }^{19}$ The dismutation rate greatly increases with the $\mathrm{O}_{2}{ }^{--}$concentration because it is a second-order reaction, and is highly dependent on the reaction media (very fast at weakly acid or neutral $\mathrm{pH}$, characteristic for biologic systems and catalyzed by traces of metals). ${ }^{20}$ The detection principles of the antioxidant capacity are based on the quantification of the $\mathrm{O}_{2}{ }^{-21}$ or hydrogen peroxide. ${ }^{22}$

Various electrochemical methods for the quantification of the $\mathrm{O}_{2}{ }^{--}$or hydrogen peroxide produced by the dismutation of $\mathrm{O}_{2}{ }^{-}$ are available, and can be adapted to evaluate the antioxidant capacity. The electrochemical methods developed for the detection of $\mathrm{O}_{2}{ }^{--}$are based on different redox proteins (i.e. cytochrome $c$ at $+150 \mathrm{mV},{ }^{21}$ azurin at $+500 \mathrm{mV},,^{23}$ or hemin at $+150 \mathrm{mV}),{ }^{24}$ triple potential-step chronoamperometry with platinized carbon microelectrodes for discrimination between different radicals ${ }^{25}$ or superoxide dismutase that can be used either for the direct transfer of electrons ${ }^{26}$ or to catalyze the $\mathrm{O}_{2}{ }^{-}$ dismutation to hydrogen peroxide, which is subsequently quantified. ${ }^{27}$ Nevertheless, the detection of $\mathrm{O}_{2}{ }^{--}$in complex 
samples with in presence of easily oxidisable substances is difficult, and sometimes even the true origin of the analytical signal is uncertain. Thus, $70 \%$ of the current recorded with the popular superoxide biosensor based on cytochrome $c$ is due to the direct oxidation of $\mathrm{O}_{2}{ }^{--}$on the electrode without the implication of cytochrome $c .{ }^{28}$ Amperometric biosensors based on hydrogen peroxide quantification that operate in the oxidation mode are prone to interferences produced by easily oxidizable substances, some of them being exactly the analyzed antioxidants ${ }^{29}$ or uric acid $^{30}$ that is enzymatically produced during $\mathrm{O}_{2}{ }^{--}$generation. The detection of antioxidants by amperometry at high potentials was reported: $+650 \mathrm{mV}$ with a platinum electrode and a semi-permeable cellulose acetate membrane $^{27}$ or $+700 \mathrm{mV}$ platinum functionalized with a permselective poly- $m$-phenylenediamine membrane. ${ }^{22}$ Bienzymatic biosensors used for antioxidants detection were reported by the combination of XOD with a second enzyme, such as: (i) superoxide dismutase for the dismutation of $\mathrm{O}_{2}{ }^{-27}$ or (ii) catalase to remove the hydrogen peroxide co-produced by XOD and avoid interferences in the detection of $\mathrm{O}_{2}{ }^{--}$with electrodes based on cytochrome $c .{ }^{21}$ Bienzymatic systems are more complicated, expensive and difficult to operate. The need for superoxide dismutase in the case of hydrogen peroxide detection is not always justified, because $\mathrm{O}_{2}{ }^{--}$spontaneously dismutates and produces hydrogen peroxide at a fast rate. ${ }^{20}$ The use of catalase does not allow evaluating the entire antioxidant capacity against both $\mathrm{O}_{2}{ }^{--}$and hydrogen peroxide. The reaction between ascorbic acid and hydrogen peroxide was used to amperometrically evaluate the antioxidant capacity without the production of free radicals. ${ }^{31}$ However, this approach has limited applicability because hydrogen peroxide is poorly reactive in the absence of transition metal ions and is ubiquitously present in biological media. ${ }^{32}$ Nevertheless, hydrogen peroxide also induces cell death, ${ }^{33}$ and a total antioxidant capacity should investigate the protection against both radicals and hydrogen peroxide. Another alternative available for the detection of hydrogen peroxide is to operate in reduction mode at low overpotentials using horseradish peroxidase ${ }^{34}$ or peroxidase mimicking mediators, such as Prussian blue. ${ }^{31}$ The antioxidants may be detected either in batch or flow analysis systems to better control the reactions using microchannel electrodes ${ }^{35}$ or the XOD immobilized in a reactor. ${ }^{36}$

In this paper, we present an XOD based sensor for evaluating the antioxidant capacity that operates in the reduction mode with low interferences. Analytical signal quantification is based on the detection of hydrogen peroxide that is produced by the spontaneous dismutation of $\mathrm{O}_{2}{ }^{--}$. Our proposed antioxidant capacity evaluation provides physiological relevant results, since the antioxidants interact with both $\mathrm{O}_{2}{ }^{--}$and hydrogen peroxide; the remaining hydrogen peroxide that is measured is representative for the overall reactions (Fig. 1). An important advantage of the hydrogen peroxide quantification in the reduction mode, at low potentials using electrodes modified with Prussian blue, is the simplicity of the proposed detection mode, which does not require semi-selective membranes, supplementary enzymes, such as superoxide dismutase, catalase or peroxidase nor redox protein, such as cytochrome $c$. XOD biosensors in the reduction mode have been previously used for the detection of the (hypo)xanthine and the evaluation of meat freshness. ${ }^{37}$ Only one study reported antioxidant detection in the reduction mode in a bienzymatic system coupled with horseradish peroxidase, but the IC50 was $189 \mu \mathrm{M}$ ascorbic acid -a value, which is 19 times higher compared with our sensor due to a combination of factors, such as the use of immobilized XOD in a reactor, a higher substrate concentration, a shorter
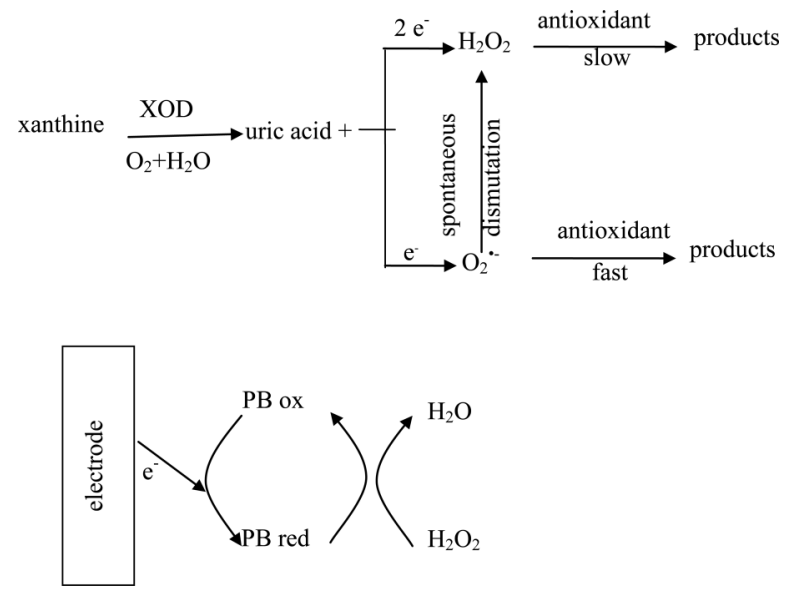

Fig. 1 Detection principle of the antioxidant capacity using amperometric quantification of hydrogen peroxide in the reduction mode. PB is Prussian blue.

reaction time and different enzymatic activity used. ${ }^{36}$ More, a complicated bienzymatic biosensor system has inherent disadvantages, e.g. the choice of a compromise $\mathrm{pH}$ between 6.0 , optimum for peroxidase and 7.5, optimum for xanthine oxidase. We have used a simple flow injection analysis (FIA) system for detection.

\section{Experimental}

\section{Reagents}

Xanthine oxidase microbial (XOD; 7.5 U/mg solid) provided by Sigma was dissolved in a phosphate buffer solution and stored at $+4^{\circ} \mathrm{C}$ in a refrigerator for up to one month. Working enzyme dilutions were prepared daily and stored under ice. A stock solution of $20 \mathrm{mM}$ xanthine (Sigma) was daily prepared in $0.1 \mathrm{M} \mathrm{NaOH}$. Stock solutions of ascorbic acid and gallic acid were prepared in a phosphate buffer, and ethanol was used for hesperetin, catechin and quercetin. A phosphate buffer solution, $0.05 \mathrm{M}$ (PBS), with $\mathrm{pH} 7.3$ supplemented with $\mathrm{KCl}$ $0.1 \mathrm{M}$ was prepared with Milli-Q ultrapure water (Millipore). Hydrogen peroxide (30\%), citric acid, sodium benzoate, glucose, aspartame and fructose were obtained from Sigma.

\section{Apparatus}

Cyclic voltammetry (CV) and amperometric measurements were performed with a galvanostat/potentiostat Autolab PGSTAT302N (Metrohm-Autolab) controlled by a PC using the software Nova 1.8. CV was made between -0.4 and $0.8 \mathrm{~V}$ at a scan rate of $100 \mathrm{mV} / \mathrm{s}$ in $\mathrm{PBS}$, in a $1 \mathrm{mM} \mathrm{H}_{2} \mathrm{O}_{2}$ solution and a mixture of $1 \mathrm{mM}$ antioxidant and $1 \mathrm{mM} \mathrm{H} \mathrm{H}_{2}$. Amperometric measurements were performed at different potentials (depending on the WE material). Screen-printed electrodes used were produced by Dropsens (Spain) on a ceramic substrate. We have investigated different WE materials: platinum (DS-550) and carbon inks modified with cobalt-phthalocyanine (DS-410) and Prussian blue (DS-710). Amperometric measurements were made in batch and flow injection system. The batch measurements were made in $5 \mathrm{~mL}$ magnetically stirred solutions; the electromagnetic noise produced by magnetic stirring was reduced with a filter from the ECD low-current module of the Autolab potentiostat set to $1 \mathrm{~s}$. The current was measured at $3 \mathrm{~min}$ after XOD injection (the current increases 

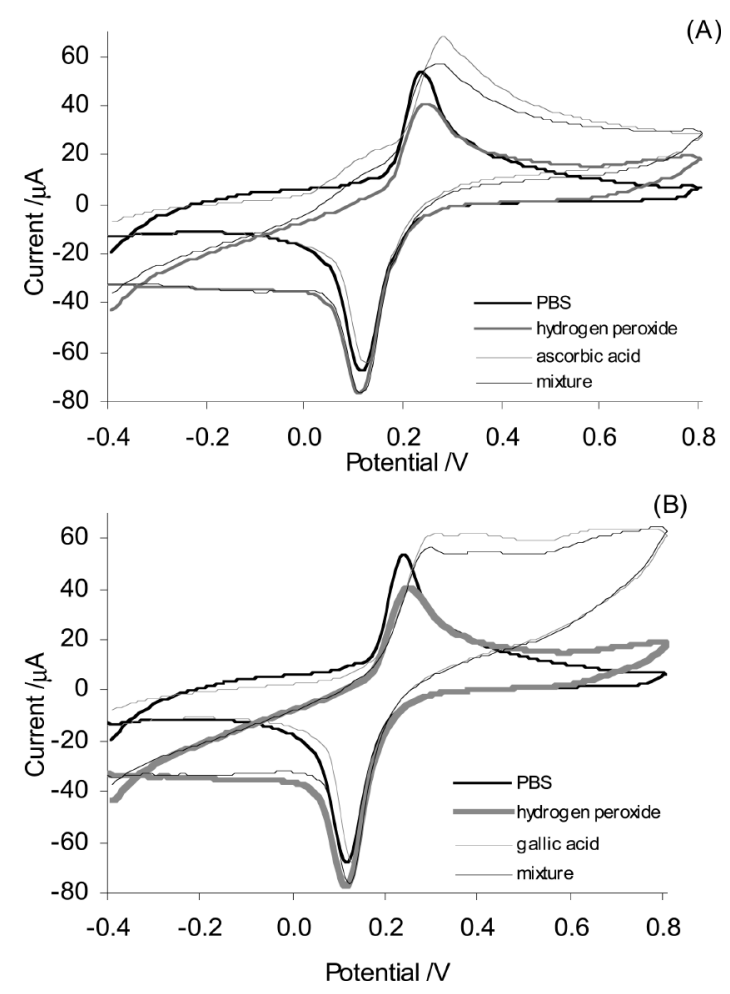

Fig. 2 Cyclic voltammograms recorded with $\mathrm{PB}$ electrodes for ascorbic acid (A) and gallic acid (B) in comparison with buffer, hydrogen peroxide and a mixture of hydrogen peroxide with an antioxidant.

linearly with time). For flow injection (FIA) system was made with a peristaltic pump Minipuls 3 (Gilson), an injection valve with a $50-\mu \mathrm{L}$ sample loop, PTFE tubing from Omnifit and a methacrylate wall jet flow cell (FLWCL from Dropsens). The optimum flow rate of PBS and xanthine was $0.15 \mathrm{~mL} / \mathrm{min}$. The filter from the potentiostat was turned off in flow measurements because there was no electromagnetic noise. For both batch and FIA measurements it was used a concentration of $30 \mu \mathrm{M}$ xanthine; $50 \mathrm{mUI}$ XOD was injected in batch and $30 \mathrm{mIU}$ XOD was injected in FIA. All the measurements were made at room temperature.

\section{Results and Discussion}

\section{Choice of the electrochemical working conditions}

Different electrochemical working conditions were investigated by $\mathrm{CV}$ and amperometry using three screen-printed working electrodes: platinum and carbon inks modified with cobalt-phthalocyanine and Prussian blue to compare the quantification of hydrogen peroxide in the oxidation and reduction modes. Five antioxidants were investigated: ascorbic acid, hesperetin, catechin, quercetin and gallic acid together with uric acid, which is both an antioxidant and a product of the enzymatic reaction. The CV studies carried out in PBS, $1 \mathrm{mM}$ hydrogen peroxide, $1 \mathrm{mM}$ antioxidant and a mixtue of $1 \mathrm{mM}$ hydrogen peroxide with $1 \mathrm{mM}$ antioxidant. Figure 2 presents data for ascorbic and gallic acids using PB electrodes, the signals being also similar for hesperetin, catechin and quercetin (see Fig. S-1, Supporting Information). The reversible peaks obtained in PBS are characteristic for PB; the CV for hydrogen peroxide shows a catalytic current in reduction domain, the $\mathrm{CV}$

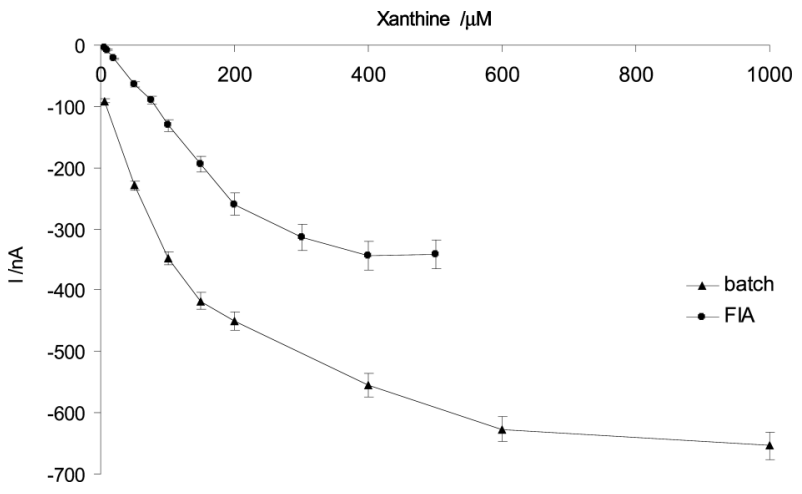

Fig. 3 Calibration plots for xanthine at a Prussian blue screenprinted electrode in batch and FIA.

for antioxidant present a current modification only in the oxidation domain; while in reduction the current is not significantly changed in comparison with PBS. The CV recorded with $\mathrm{PB}$ electrodes for hydrogen peroxide overlap in the reduction domain with the $\mathrm{CV}$ recorded for hydrogen peroxide mixed with an antioxidant, indicating the possibility to detect hydrogen peroxide in presence of antioxidants. Both platinum and cobalt-phthalocyanine electrodes may detect hydrogen peroxide in the oxidation mode only at high potentials $(+600 \mathrm{mV})$, but the oxidation currents recorded for all investigated antioxidants are very high and prevent their use (data not shown). These results demonstrated that only Prussian blue electrodes operated in the reduction mode allow the quantification of hydrogen peroxide in the presence of antioxidants.

Amperometric measurements were made in batch using magnetically stirred solutions at $+600 \mathrm{mV}$ for platinum and cobalt-phthalocyanine electrodes and $-50 \mathrm{mV}$ for Prussian blue electrodes. The amperometric current was measured after $20 \mathrm{~s}$. The interfering signals were first evaluated by successive injections of 5,10 and $20 \mu \mathrm{M}$ of each antioxidant in PBS. Another strategy consisted of injecting the same concentrations of the antioxidants in 10,20 or $50 \mu \mathrm{M}$ hydrogen peroxide standard solutions. For Prussian blue electrodes it was obtained a small interfering signal only from ascorbic acid (i.e. for the injection of $15 \mu \mathrm{M}$ ascorbic acid the interfering signal is smaller than $18 \mathrm{nA}$, while the signal recorded for enzymatic measurements is higher than $-100 \mathrm{nA}$, even for low substrate concentrations). The injection of five other antioxidants (hesperetin, catechin, quercetin, gallic acid and uric acid) did not produce a significant amperometric signal for the Prussian blue electrodes. Both platinum and cobalt-phthalocyanine electrodes had high interfering signals that prevented their use (i.e. the signal obtained from the injection of $15 \mu \mathrm{M}$ uric acid was $+1098 \mathrm{nA}$ for platinum and $+1114 \mathrm{nA}$ for cobaltphthalocyanine electrodes).

\section{Optimization of the antioxidant capacity evaluation}

The initial optimization was carried out in batch with the enzyme being injected into the solution. The minimum enzyme activity necessary to obtain a significant analytical signal was chosen to be $50 \mathrm{mIU}$ using $1 \mathrm{mM}$ xanthine (saturation with substrate).

The calibration graph for the enzymatic substrate is linear between $5-150 \mu \mathrm{M}$ xanthine (Fig. 3), and the plateau starts at around $600 \mu \mathrm{M}$. A high concentration of the substrate is usually chosen for measurements with enzymatic sensors based on 


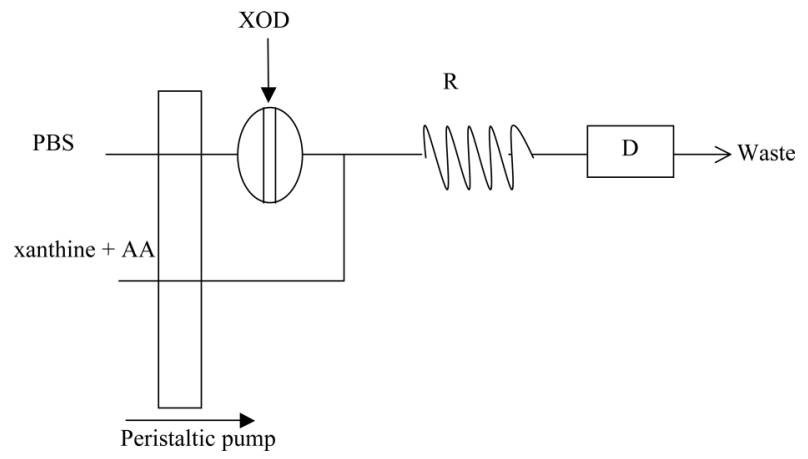

Fig. 4 Schematic diagram of the FIA system. PBS-phosphate buffer, AA-antioxidant or real sample, R-reactor, D-detector (electrode in a flow-cell).

signal reduction by the analyte in order to have a maximum initial analytical signal, and to use a minimum enzyme quantity. In our case, the concentration of the enzymatically produced $\mathrm{O}_{2}{ }^{--}$in the reaction medium must be optimized for the antioxidant capacity evaluation. A higher $\mathrm{O}_{2}{ }^{--}$concentration is produced with higher xanthine concentrations, but this implies a faster spontaneous dismutation of the $\mathrm{O}_{2}{ }^{--}$into hydrogen peroxide and oxygen without the radical reaction with antioxidants. The spontaneous dismutation of $\mathrm{O}_{2}{ }^{--}$is a secondorder reaction, therefore the radical lifetime is highly dependent on its initial concentration. ${ }^{20,38}$ On the other hand, the xanthine concentration must be sufficient to provide an analytical signal with a magnitude that allows the quantification of the signal reduction induced by the antioxidant. For antioxidant detection, we have used a lower xanthine concentration (to reduce the $\mathrm{O}_{2}{ }^{-}$ concentration and its rate of spontaneous dismutation) coupled with a longer reaction time (to increase the total quantity of $\mathrm{O}_{2}{ }^{--}$that is produced). Nevertheless, antioxidants react with both $\mathrm{O}_{2}{ }^{--}$and hydrogen peroxide, the optimization of the xanthine concentration being made to avoid the premature dismutation of $\mathrm{O}_{2}{ }^{--}$. The optimum xanthine concentration was chosen by investigating the reduction of the analytic signal produced by the same concentration of ascorbic acid at various substrate concentrations: $10 \mu \mathrm{M}$ ascorbic acid and $30-150 \mu \mathrm{M}$ xanthine range, respectively. The maximum reduction of the analytical signal was obtained for $30 \mu \mathrm{M}$ xanthine (a $51 \%$ decrease for $30 \mu \mathrm{M}$ xanthine, a $23.5 \%$ decrease for $50 \mu \mathrm{M}$ xanthine and only $15.6 \%$ for $100 \mu \mathrm{M}$ xanthine). Consequently, the concentration of $30 \mu \mathrm{M}$ xanthine was chosen to evaluate the antioxidant capacity. Another advantage of the low substrate concentration usage is to minimize the uric acid production, thus avoiding the apparition of a new antioxidant into the reaction media.

Optimization of the antioxidant capacity evaluation using the FIA system

The flow injection system is presented in Fig. 4 and consists of the two flows: (1) substrate or substrate with the antioxidant and (2) a PBS solution. The enzyme was injected into the PBS flow using the injection valve, and then merged with the second flow in a reactor that ensures a sufficient time for $\mathrm{O}_{2}{ }^{-}$production. The $\mathrm{O}_{2}{ }^{--}$is simultaneously consumed by the antioxidants, or dismutates into oxygen and hydrogen peroxide, which provides the analytical signal.

Different working parameters were optimized: the concentration of substrate, the enzyme activity, the tubes length,
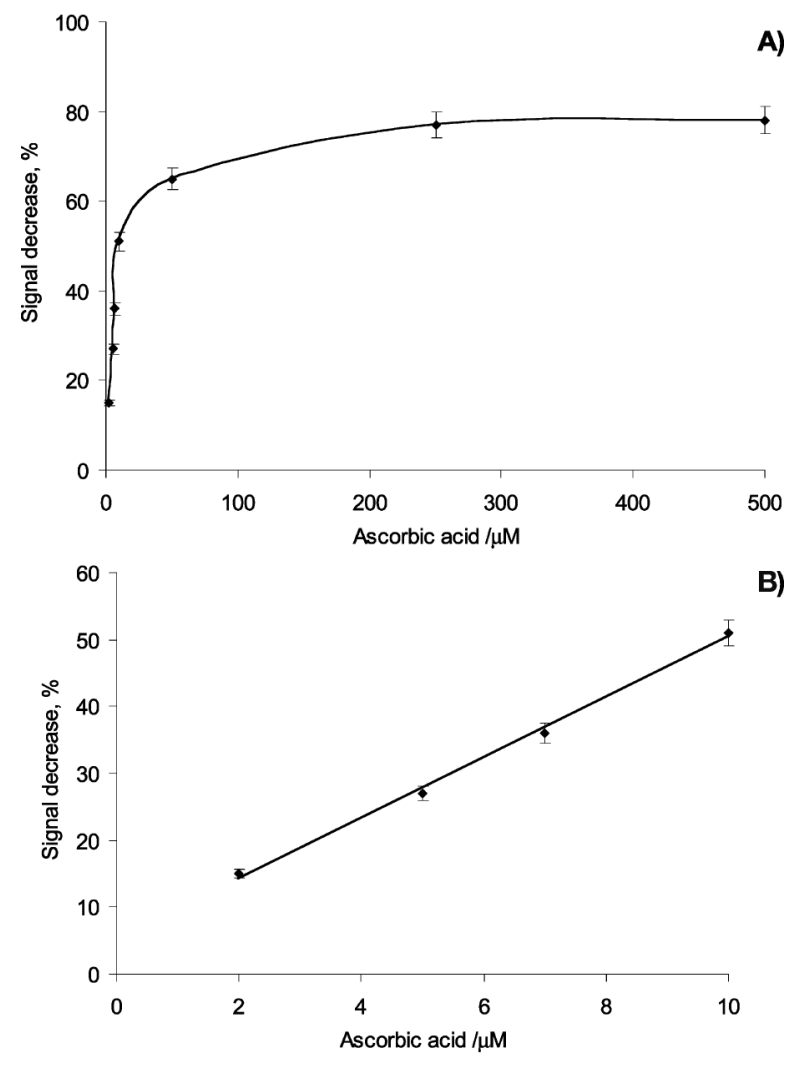

Fig. 5 Calibration plot for ascorbic acid obtained with a Prussian blue screen-printed electrode in flow injection analysis. A) Entire domain; B) linear part.

and the flow rate. The flow rate in combination with the reactor length determines both the reaction time and the injected enzyme dispersion (broadening and flattening of the FIA peaks). A longer reaction time leads to the production of a higher quantity of $\mathrm{O}_{2}{ }^{-}$, corresponding to higher analytical signals, but also an increased dispersion. The effect of the flow rate on the analytical signal was studied in $0.1-1.5 \mathrm{~mL} / \mathrm{min}$ range and the reactor length was varied between 25 and $110 \mathrm{~cm}$. The optimum conditions determined from these studies were: the reactor length $(105 \mathrm{~cm})$ and the total flow rate $(0.3 \mathrm{~mL} / \mathrm{min})$. The long residence time in the reactor $(5 \mathrm{~min})$ does not imply a long analysis time, because several injections can be made sequentially, and reach the detector one after another (one analysis is made in less than $3 \mathrm{~min}$ ). The optimum enzyme activity to obtain a significant analytical signal was $22 \mathrm{mUI}$, a lower value compared with batch measurements. The calibration graph for xanthine is linear between $5-200 \mu \mathrm{M}$; the plateau was reached at $400 \mu \mathrm{M}$ (Figs. 3 and S-2 (Supporting Information) for the analytical signals). The optimum substrate concentration for evaluating the antioxidant capacity was established at $30 \mu \mathrm{M}$, and was determined by the decrease of the analytical signal produced by $10 \mu \mathrm{M}$ ascorbic acid.

The ascorbic acid was used as a model compound for the estimation of antioxidant capacity. Standard solutions with different concentrations of ascorbic acid were mixed with $30 \mu \mathrm{M}$ xanthine, and analyzed using the FIA system. A decrease of the analytical signal correlated with the ascorbic acid concentration, which is attributed to the consumption of both $\mathrm{O}_{2}{ }^{--}$and hydrogen peroxide by the antioxidant. By plotting the percentage decrease $(\%)$ of the substrate signal $v s$. the ascorbic acid concentration, we obtained a linear calibration graph 
between $2-10 \mu \mathrm{M}$, followed by a large plateau for higher concentrations of the antioxidant (Fig. 5). The decrease of the analytical signal obtained at $10 \mu \mathrm{M}$ ascorbic acid was $50 \%$. The maximum decrease of the analytical signal was $80 \%$ even at very high antioxidant concentrations: $250-500 \mu \mathrm{M}$ ascorbic acid (i.e. a concentration that is 50 times greater compared with the linear part of the calibration graph). This is explained by the kinetics of the overall reaction mechanism: $\mathrm{O}_{2}{ }^{--}$reacts fast with antioxidants, while hydrogen peroxide is consumed at a much slower rate by the antioxidant. In fact, hydrogen peroxide is poorly reactive in the absence of transition metal ions and is present in cell cultures. ${ }^{32}$

The detection limit of the enzymatic sensor was $0.8 \mu \mathrm{M}$. The limit of detection was calculated using the formula LOD = $(3 \times \mathrm{sb}) / \mathrm{S}$, where $\mathrm{sb}$ represents the standard deviation of the blank signal, and $S$ represents the sensitivity. The reproducibility of the substrate measurements was $6.9 \%$ for the batch system and $3.3 \%$ for FIA system. The reproducibility of the antioxidant measurements carried out in batch and in flow were calculated and found to be 5.8 and $3.9 \%$, respectively.

\section{Interferences}

The sensor is selective towards easily oxidisable substances due to measurements at low potential in the reduction mode. Other substances usually found in foods (citric acid, sodium benzoate, glucose, aspartame and fructose) were studied as possible interfering substances. First, the interferents were injected in the PBS without XOD or xanthine in order to evaluate any eventual electrochemical interference. The interfering signals recorded for $1 \mathrm{mM}$ concentrations were lower than $-12 \mathrm{nA}$, while the analytical signal was $-65 \mathrm{nA}$. The influence of the interferents was also studied by mixing the interferent (from $25 \mu \mathrm{M}$ to $1 \mathrm{mM}$ ) with xanthine $(30 \mu \mathrm{M})$ or xanthine $(30 \mu \mathrm{M})$ and ascorbic acid $(10 \mu \mathrm{M})$. The results showed that all tested compounds had no interference for concentrations lower than $500 \mu \mathrm{M}$.

\section{Real sample analysis}

The developed sensor based on XOD and mediated amperometric measurements in the reduction mode was used to evaluate the antioxidant capacity in different juices real samples. Non-carbonated juices with added ascorbic acid were purchased from a local supermarket. Fresh lemon and orange juices were prepared from fruits before analysis. The real samples were homogenized and then filtered. Then, $100 \mu \mathrm{L}$ of the filtered sample were diluted to $10 \mathrm{~mL}$ with a solution of $30 \mu \mathrm{M}$ xanthine prepared in a buffer and analyzed as described in the FIA procedure. The results obtained for the determination of the total antioxidant capacity in fresh orange and lemon juices are comparable to those reported in the literature. ${ }^{39}$ For the other real samples, the concentration of the added ascorbic acid was not mentioned on the label. A recovery test was made for a concentration of $10 \mu \mathrm{M}$ ascorbic acid added to each sample; the results were found to be between $94.3-103.1 \%$. The results are presented in the Table 1.

\section{Conclusions}

A sensor based on xanthine oxidase was developed to evaluate the antioxidant capacity with low interferences using mediated amperometric measurements in the reduction mode. No interference was observed from easily oxidisable substances, or other compounds usually found in food samples. The system is simple and does not require a second enzyme or other
Table 1 Real sample analysis

\begin{tabular}{lcc}
\hline \multicolumn{1}{c}{ Sample } & $\begin{array}{c}\text { mg equivalent } \\
\text { ascorbic } \\
\text { acid/1 L } \\
\text { sample } \pm \text { SD }\end{array}$ & $\begin{array}{c}\text { Recovery of } \\
\text { the spiked } \\
\text { ascorbic acid, } \\
\%\end{array}$ \\
\hline Orange juice 12\% & $299 \pm 9$ & 97.2 \\
Orange juice min. 7\% & $528 \pm 12$ & 94.3 \\
Fruits juice 12\% & $880 \pm 24$ & 103.1 \\
$\quad$ (carrots-red oranges-limes-apples) & & \\
Strawberries-apples juice & $827 \pm 26$ & 102.2 \\
Lemon tea & $385 \pm 12$ & 97.6 \\
Fresh lemon juice & $574 \pm 20$ & 95.7 \\
Fresh orange juice & $498 \pm 16$ & 94.9 \\
\hline
\end{tabular}

mechanisms for the minimization of interferences. The measurements were made by using an FIA system coupled with a reactor that ensured a sufficient time for the reproductively production of the superoxide radical $\left(\mathrm{O}_{2}{ }^{--}\right)$at low concentrations in order to minimize the spontaneous dismutation of the free radical. Importantly, our system is able to evaluate the real antioxidant capacity of food samples by their interaction with both $\mathrm{O}_{2}{ }^{-}$and hydrogen peroxide to provides physiologically relevant results. This paper also provides a simple and reliable method for investigating the complex enzymatic reactions of xanthine oxidase (XOD).

\section{Acknowledgements}

This work was supported by grants of the Romanian Department of Education and Research (MEN-UEFISCDI) projects: PN-IIRU-TE-2011-3-0076 and PN-II-PT-PCCA-2013-4-0203.

\section{References}

1. J. Viña, C. Borras, K. M. Abdelaziz, R. Garcia-Valles, and M. C. Gomez-Cabrera, Antioxid. Redox. Signaling, 2013, 19, 779 .

2. E. Baudouin, Plant. Biol., 2011, 13, 233.

3. M. Gutowski and S. Kowalczyk, Acta Biochim. Pol., 2013, $60,1$.

4. G. Bartosz, Free Radical Res., 2010, 44, 711.

5. P. Detopoulou, D. B. Panagiotakos, C. Chrysohoou, E. Fragopoulou, T. Nomikos, S. Antonopoulou, C. Pitsavos, and C. Stefanadis, Eur. J. Clin. Nutr., 2010, 64, 161.

6. EFSA Panel on Dietetic Products, Nutrition and Allergies (NDA), EFSA J., 2010, 821489.

7. United States Department of Agriculture, http://www.ars. usda.gov/Services/docs.htm?docid=15866.

8. I. Binic, V. Lazarevic, M. Ljubenovic, J. Mojsa, and D. Sokolovic, J. Evidence-Based Complementary Alternat. Med., 2013, 2013, 1

9. T. Shimamura, Y. Sumikura, T. Yamazaki, A. Tada, T. Kashiwagi, H. Ishikawa, T. Matsui, N. Sugimoto, H. Akiyama, and H. Ukeda, Anal. Sci., 2014, 14, 717.

10. B. Shen, J. Truong, R. Helliwell, S. Govindaraghavan, and N. J. Sucher, BMC Complementary Altern. Med., 2013, 13, 373.

11. K.-C. Cheng, J.-Y. Wu, J.-T. Lin, and W.-H. Liu, Eur. Food Res. Technol., 2013, 236, 1107.

12. M. Barontini, R. Bernini, I. Carastro, P. Gentili, and 
A. Romani, New J. Chem., 2014, 38, 809.

13. A. Bast and G. R. M. M. Haenen, Trends Pharmacol. Sci., 2013, 34, 430.

14. Y. Ye, J. Li, and Z. Yuan, PLoS One, 2013, 8, e56803.

15. M. E. Martinez, E. T. Jacobs, J. A. Baron, J. R. Marshall, and T. Byers, J. Natl. Cancer Inst., 2012, 104, 732.

16. X. Xin, R. Fan, Y. Gong, F. Yuan, and Y. Gao, Eur. Food Res. Technol., 2014, $238,837$.

17. B. Prieto-Simón, M. Cortina, M. Campàs, and C. CalasBlanchard Sens. Actuators, B, 2008, 129, 459.

18. J.-K. Moon, and T. Shibamoto, J. Agric. Food Chem., 2009, 57,1655

19. S. Ignatov, D. Shishniashvili, B. Ge, F. W. Scheller, and F. Lisdat, Biosens. Bioelectron., 2002, 17, 191.

20. B. H. J. Bielski, D. E. Cabelli, R. L. Arudi, and A. B. Ross, J. Phys. Chem. Ref. Data, 1985, 14, 1041.

21. M. Cortina-Puig, X. Muñoz-Berbel, C. Calas-Blanchard, and J. L. Marty, Talanta, 2009, 79, 289.

22. M. Cortina-Puig, A. C. H. Scangas, Z. S. Marchese, S. Andreescu, J. L. Marty, and C. Calas-Blanchard, Electroanalysis, 2010, 22, 2429.

23. S. Shleev, J. Wetterö, K. E. Magnusson, and T. Ruzgas, Biosens. Bioelectron., 2006, 22, 213.

24. J. Chen, U. Wollenberger, F. Lisdat, B. Ge, and F. W. Scheller, Sens. Actuators, B, 2000, 70, 115.

25. C. Amatore, S. Arbault, and A. C. W Koh, Anal. Chem., 2010, 82, 1411.

26. A. Kapp, M. K. Beissenhirtz, F. Geyer, F. Scheller, M. S. Viezzoli, and F. Lisdat, Electroanalysis, 2006, 18, 1909.
27. L. Campanella, G. Favero, and M. Tomassetti, Anal. Lett., 1999, 32, 2559.

28. S. Gáspár, J. L. Marty, and E. Gheorghiu, Electroanalysis, 2013, 25, 448

29. B. Bucur, G. L. Radu, and C. N. Toader, Eur. Food Res. Technol., 2007, 226, 1335.

30. B. N. Ames, R. Cathcart, E. Schwiers, and P. Hochstein, Proc. Natl. Acad. Sci. U. S. A., 1981, 78, 6858.

31. E. E. Karyakina, D. V. Vokhmyanina, N. V. Sizova, A. N. Sabitov, A. V. Borisova, T. G. Sazontova, Y. V Arkhipenko, V. A. Tkachuk, Y. A. Zolotov, and A. A. Karyakin, Talanta, 2009, 80, 749.

32. B. Halliwell, M. V. Clement, J. Ramalingam, and L. H. Long, IUBMB Life, 2000, 50, 251.

33. D. Liu and F. Bao, Neuroscience, 2015, 285, 81.

34. F. Chekin, L. Gorton, and I. Tapsobea, Anal. Bioanal. Chem., 2015, 407, 439.

35. R. Oliveira, F. Bento, C. Sella, L. Thouin, and C. Amatore, Anal. Chem., 2013, 85, 9057.

36. V. Lates, J. L. Marty, and I. C. Popescu, Electroanalysis, 2010, 23, 728.

37. N. Dolmac1, S. Çete, F. Arslan, and A. Yaşar, Artif. Cells, Blood Substitutes, Biotechnol., 2012, 40, 275.

38. I. Fridovich, Annu. Rev. Pharmacol. Toxicol., 1983, 23, 239.

39. Y. S. Parka, M. H. Imb, K. S. Hamc, S. G. Kangc, Y. K. Parkc, J. Namiesnikd, H. Leontowicze, M. Leontowicze, S. Trakhtenbergf, and S. Gorinsteing, LWT-Food Sci. Technol., 2015, 63, 346. 